\title{
Solid Solution Lithium Alloy Cermet Anodes
}

\author{
Thomas J. Richardson* and Guoying Chen \\ Environmental Energy Technologies Division \\ Lawrence Berkeley National Laboratory \\ Berkeley, California 94720 USA
}

\begin{abstract}
Lithium-magnesium solid solution alloys with compositions between $\mathrm{Li}_{0.6} \mathrm{Mg}_{0.4}$ and $\mathrm{Li}_{0.8} \mathrm{Mg}_{0.2}$ were prepared by melting the component metals in argon. Experiments carried out in a transparent cell confirmed the suppression of dendrite formation on the alloy surface. Diffusion kinetics within the bulk alloy limit the practical current density, particularly during discharging. Heating mixtures of lithium nitride and magnesium provides a convenient method of preparing ceramic-metal composites ("cermets") containing the solid solution alloy and inert magnesium nitride. The cermets can be formed into a desired shape before or after reaction and may offer a route to higher surface area metallic anodes with improved rate capability.
\end{abstract}

\section{Introduction}

Lithium alloys such as Li-Sn and Li-Si are attractive candidates to replace graphite due to their very large theoretical capacities $[1,2]$. In practice, however, the binary materials suffer from significant irreversible capacities, poor cyclability, and questionable rate capability. These problems arise from the complexity of their phase diagrams and the fact that substantial atomic rearrangements and large volume changes accompany a series of first-order phase transitions. This results in exposure of fresh surfaces to the electrolyte, disconnection and isolation of active material, and inhomogeneity within the composite electrode. An electrode in which a solid solution of lithium and another element exists over a wide composition range would be highly advantageous. Charging and discharging result in continuous and relatively stress-free volume changes similar to those in a well-behaved lithium foil electrode, thus reducing irreversible capacity losses and electrolyte consumption. More importantly, a solid solution exhibits a sloping potential vs. composition profile, which reflects the presence of a driving force for relaxation to a uniform composition. Given sufficient mobility in the alloy, lithium would be transported away from the surface during deposition, countering the tendency to form dendrites.

Few examples of such solid solutions with significant capacities are known. They appear in very limited composition ranges in the $\mathrm{Li}-\mathrm{Sn}$ and $\mathrm{Li}-\mathrm{Si}$ systems. ( $\mathrm{LiHg}$ ) appears as a single phase over the range from $38 \%$ to $62 \%$ lithium [3], but this corresponds to only $129 \mathrm{mAh} / \mathrm{g}$. Even smaller ranges exist for indium and cadmium. The most attractive is the lithiummagnesium system, in which a solid solution with the bcc lithium structure covers the entire range from $30 \%$ to nearly $100 \%$ lithium. Utilizing the range from $40 \%$ to $70 \% \mathrm{Li}$, for example, would give a capacity of $1100 \mathrm{mAh} / \mathrm{g}$. Li-Mg alloy anodes have been described in several patents [4-7], but are rarely mentioned in the open literature. The system was investigated recently by Shi et al. [8], who reported high mobility for lithium in the solution phase, but this did not translate into superior electrode performance.
Here we describe lithium plating and stripping experiments in a transparent cell in which the tendency toward dendrite formation on a pure lithium surface is compared with that of a foil containing 30 at. $\% \mathrm{Mg}$. Preparation of $\mathrm{Li}-\mathrm{Mg}$ alloy cermets by a simple metathesis reaction is also reported.

\section{Experimental}

Lithium-magnesium alloys were prepared by melting lithium foil and magnesium powder or ribbon in a steel crucible in an argon atmosphere. Alloys with $\mathrm{Mg}$ content up to $30 \%$ were melted in an open crucible furnace in a controlled atmosphere glove box. Metal mixtures with higher Mg content were placed in a tantalum crucible and sealed in a copper-gasketed stainless steel container, then heated in a box furnace. The alloy ingots were cold-rolled into foils between spring steel sheets to prevent adhesion to the rollers.

The transparent cells for visual observation of electrode surfaces during cycling were similar to those described by Howlett et al. [9]. $\mathrm{Li}$ and/or $\mathrm{Li}_{0.7} \mathrm{Mg}_{0.3}$ foils (about $0.3 \mathrm{~mm}$ thick, $4 \mathrm{~mm}$ wide and $10 \mathrm{~mm}$ long) were pressed onto copper current collectors placed on top of a glass slide. The distance between the electrodes was $1 \mathrm{~mm}$. A piece of polyvinylidene difluoride (PVDF) membrane (1 mm x $4 \mathrm{~mm}$, Gelman FP Vericel) wetted by $1 \mathrm{M} \mathrm{LiClO}_{4}$ in propylene carbonate was placed between the electrodes so that only the opposing edges were in contact with the electrolyte. Another glass plate was laid on the top of the assembly, and the cell was sealed with low vapor pressure epoxy (Torr-seal, Varian Associates) before removal from the glove box. Galvanostatic charge-discharge cycling was performed using a Gamry potentiostat. Each cycle consisted of a charge and discharge of $3 \mathrm{C} / \mathrm{cm}^{2}$ at a current density of $2 \mathrm{~mA} / \mathrm{cm}^{2}$ ( $25 \mathrm{~min}$ ). Images were collected using a CCD camera coupled to an optical microscope.

$\mathrm{Mg}_{3} \mathrm{~N}_{2}$-LiMg cermets were prepared by mixing $\mathrm{Li}_{3} \mathrm{~N}$ powder $(<150 \mu \mathrm{m})$ with $\mathrm{Mg}$ powder $(<45 \mu \mathrm{m})$ according to Eq. (1):

$2 \mathrm{Li}_{3} \mathrm{~N}+(3+\mathrm{x}) \mathrm{Mg} \rightarrow \mathrm{Li}_{6} \mathrm{Mg}_{\mathrm{x}}+\mathrm{Mg}_{3} \mathrm{~N}_{2}$ 
(a)

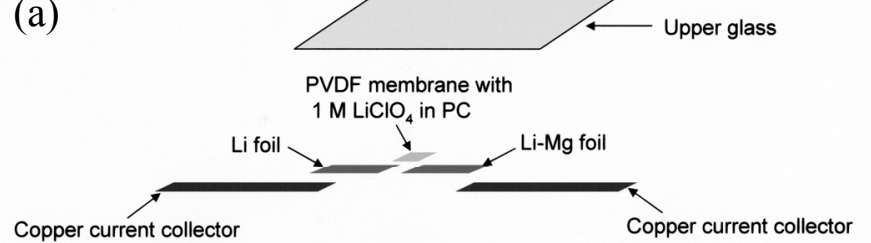

Copper current collector

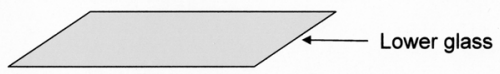

(b)

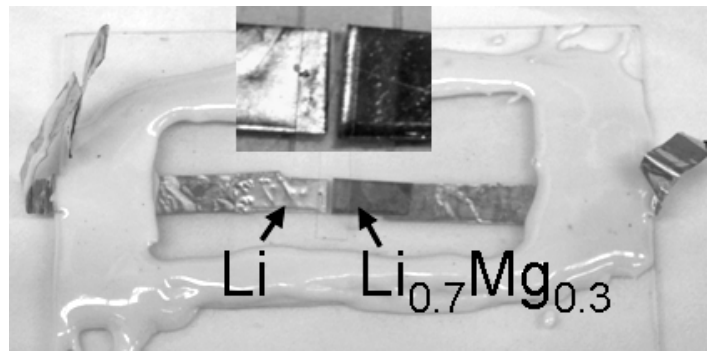

Fig. 1. Transparent cell: (a) schematic diagram, (b) photo with enlarged inset. The gap between electrodes is $1 \mathrm{~mm}$.

and heating to the melting point of the alloy phase $\left(310^{\circ}\right.$ to $480^{\circ} \mathrm{C}$ for 20 to 40 at. $\% \mathrm{Mg}$ ) in a tantalum boat. The freeflowing powder were pressed in to discs of varying porosity depending upon the applied pressure. These could be used as is or rolled into thin sheets and cut in the same manner as foils. It is also possible to press the raw powder mixture into discs, and these retained their shape after heating with some shrinkage due to sintering. X-ray diffraction patterns were obtained from pressed discs protected from reaction with air by a beryllium window. That of a cermet containing $\mathrm{Li}_{0.7} \mathrm{Mg}_{0.3}$ (51 wt. \%, 74 vol. \%) and $\mathrm{Mg}_{3} \mathrm{~N}_{2}$ is shown in Fig. 2. The peak positions for $\mathrm{Li}_{0.7} \mathrm{Mg}_{0.3}$ are slightly shifted from those of pure lithium in agreement with the reported lattice parameters [10]. A small amount of unreacted $\mathrm{Li}_{3} \mathrm{~N}$ is present, but no residual $\mathrm{Mg}$.

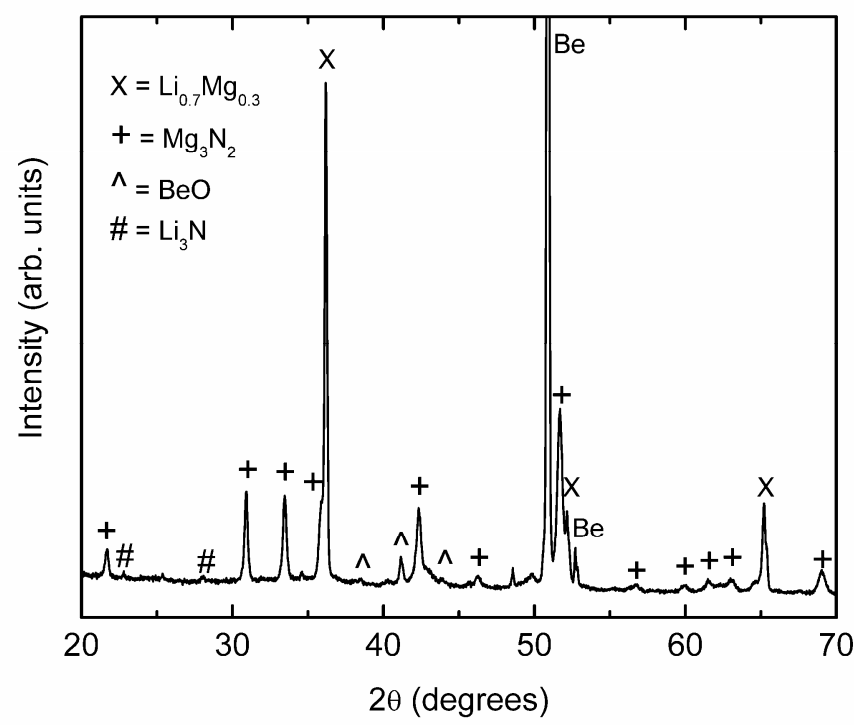

Fig. 2. X-ray powder diffraction pattern of $\mathrm{Li}_{0.7} \mathrm{Mg}_{0.3}-\mathrm{Mg}_{3} \mathrm{~N}_{2}$ cermet.

\section{Results and Discussion}

\subsection{Suppression of dendrite growth on Li-Mg}

The characteristic behavior of Li during stripping and plating in non-aqueous electrolytes is shown in Fig. 3(a). Dendrites formed during plating become disconnected from the surface during stripping and form a mass of loose particles, which is pushed away from the surface as new dendrites form behind it. This is the mechanism by which lithium cells typically become shorted, rather than by single dendrites extending across the separator. In contrast, although the Li-Mg alloy surface, shown in Fig. 3(b), becomes rougher, and nodules of significant dimensions are produced, small dendrites are not observed, and particles are not released into the electrolyte.

This may be understood by considering the state of lithium atoms freshly deposited on the alloy surface. The high concentration of lithium at the surface and the lower chemical potential of lithium within the bulk alloy (as measured by a potential difference of about $30 \mathrm{mV}$ ) provide a driving force for lithium to diffuse into the bulk. This may even result in dissolution of pure lithium from protruding features and redeposition on nearby flat surface areas with higher $\mathrm{Mg}$ content. No increase in cell impedance was observed over the course of several hundred cycles.
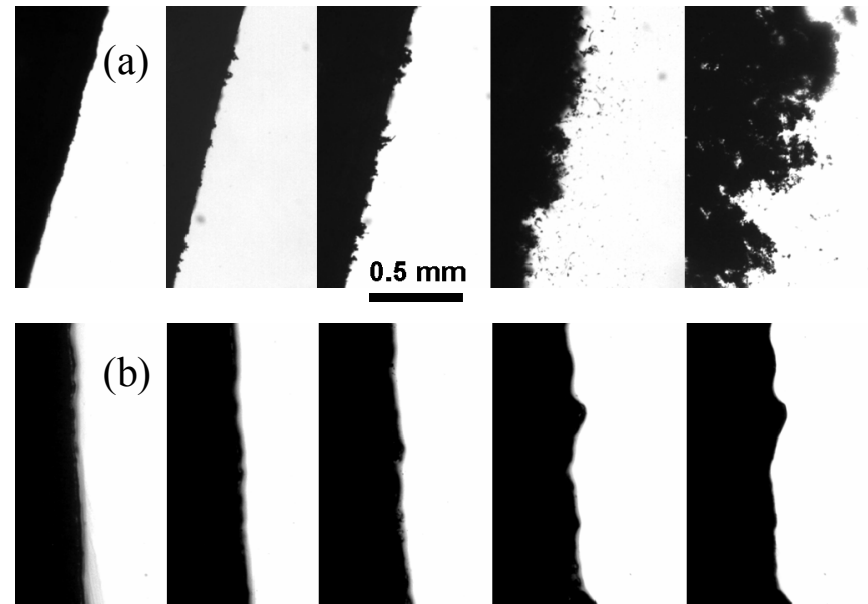

(b)
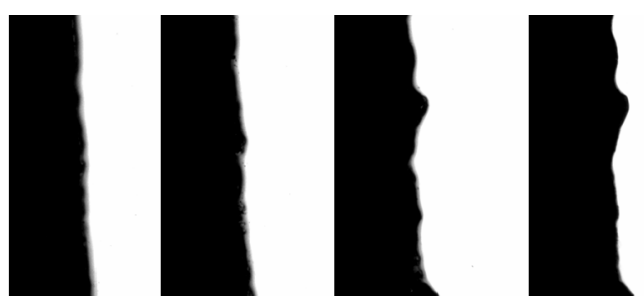

Fig. 3. Optical micrographs of (a) lithium surface and (b) $\mathrm{Li}_{0.7} \mathrm{Mg}_{0.3}$ surface following $0,16,46,94$, and 192 cycles. The counter electrode in each case was $\mathrm{Li}$ foil.

During lithium stripping at low rates, lithium diffuses toward the surface as it becomes depleted. At higher rates, however, the Li content at the surface may fall below the minimum (30 at. \%) required to maintain the bcc [Li] phase in which $\mathrm{Li}$ has high mobility [11]. Formation of the hcp [Mg] solid solution phase results in a rapid shutdown of lithium diffusion and an increase in the electrode potential. This may occur at rates as low as $0.5 \mathrm{~mA} / \mathrm{cm}^{2}$, but it varies with electrolyte concentration and cell configuration. At potentials above about $0.65 \mathrm{~V}$ vs. Li, $\mathrm{Mg}$ may be dissolved along with lithium. Although this behavior imposes a discharge rate limit that may be undesirable in a high power cell, it may provide a measure of protection against rapid discharge on inadvertent shorting of a high energy density cell. Increasing the surface area by introducing 
porosity could increase the rate capability, but a metallic electrode of this type would be susceptible to degradation due to dimensional changes during cycling.

\subsection{Li-Mg Alloy Cermets}

In preliminary cycling experiments, dense $\mathrm{Li}-\mathrm{Mg}$ cermets performed somewhat better than their foil counterparts in terms of rate capability and potential relaxation following stripping. A detailed report will be submitted in the near future.

Lithium-rich Li-Mg alloys are soft and tend to stick to rollers or protective sheets when rolled to make foils. Mg-rich alloys require high temperatures for preparation and are difficult to extract from crucibles. In addition, at each composition, the molten alloy passes through a peritectic region before it completely solidifies, resulting in precipitation of solid solution grains of varying composition. While the inhomogeneity can be removed by annealing, it causes the freshly cooled metal to fracture during rolling.

Ceramic-metal composites, or cermets, are widely used in industry (as porous electrodes in solid oxide fuel cells, for example) because they are more heat-resistant than metals, have greater fracture toughness, and can be formed by plastic deformation. They are typically made by sintering of intimate mixtures of ceramic and metal powders, but in some cases, the ceramic portion is formed by oxidation of a metallic precursor. A microporous lithium or lithium alloy cermet containing a lightweight ceramic component would have certain advantages over its metallic counterpart both in performance and manufacturing. Maintaining porosity during mixing of soft lithium metal with a ceramic powder, however, is difficult. Magnesium powder, on the other hand, mixes easily with lithium nitride, and both can be obtained as very fine powders. The ceramic component in the final product is magnesium nitride, formed by metathesis. The metallic component may be pure lithium or lithium-magnesium alloy of any desired composition.

\section{Conclusions}

Lithium-magnesium alloy electrodes maintain their physical integrity and appear to suppress the formation of dendrites during stripping and plating of lithium in a non-aqueous electrolyte. Their high lithium content and light weight make them an attractive alternative to carbon anodes. Certain rate limitations, however, must be addressed if they are to be used in high power cells.

High quality Li-Mg alloys are difficult to prepare and handle in the laboratory due to their reactivity and high melting temperatures. A convenient metathesis reaction has been developed which produces an easily formed cermet containing the alloy and a ceramic second phase. The ability to form the mixture into a desired shape prior to reaction may facilitate production of highly porous electrodes with minimal postreaction manipulation.

\section{Acknowledgement}

This work was supported by the Assistant Secretary for Energy Efficiency and Renewable Energy, Office of FreedomCAR and Vehicle Technologies of the U.S. Department of Energy under Contract No. DE-AC02-05CH11231.

\section{References}

[1] J. Yang, M. Winter, and J. O. Besenhard, Solid State Ionics 90 (1996) 281.

[2] C. J. Wen and R. A. Huggins, J. Solid State Chem. 37 (1981) 271.

[3] M. Hansen and K. Anderko, Constitution of Binary Alloys, 2nd Ed., Genium Publishing Corp., Schenectady, NY, 1989, p. 821.

[4] R. T. Mead, US Patent 3,895,962 (1975).

[5] G.-M. Raynaud, G. Regazzoni, G. Nussbaum, and M. Reboul, US Patent 5,102,475 (1992).

[6] S. F. Hope and J. B. Kejha, US Patent 5,350,647 (1994).

[7] D. O. Hobson, US Patent 5,705,293 (1998).

[8] Z. Shi, M. Liu, D. Naik, and J. L. Gole, J. Power Sources 92 (2001) 70.

[9] P. C. Howlett, D. R. MacFarlane, and A. F. Hollenkamp, J. Power Sources 114 (2003) 277.

[10] F. H. Herbstein and B. L. Averbach, Acta Metallugica 4 (1956) 407.

[11] Y. Iwadate, M. Lassouani, F. Lantelme, M. Chemla, J. Appl. Electrochem. 17 (1987) 385. 